\title{
Scope of inflammopharmacology
}

\author{
K. D. Rainsford
}

(C) Springer Basel AG 2010

Acute and chronic inflammatory processes underlie a vast number of diseases. These processes involve production of many inflammatory mediators, which are formed from or interact with a wide range of cells, including those of the immune system, to cause various responses at specific sites in the body.

This presents challenges for drug treatment of these conditions and for the effectiveness of conventional agents such as non-steroidal anti-inflammatory drugs (NSAIDs), corticosteroids, disease-modifying anti-rheumatic drugs (DMARDs), anti-cytokine and other biologic agents, as well as natural products (or nutraceuticals). The use of many of these agents is often limited by (a) the agents' unspecific actions or limited potency at their therapeutic targets, (b) their poor pharmacokinetic properties leading to poor bioavailability of the active agents and (c) their varying array of serious and non-serious side effects and adverse drug reactions.

Broadly speaking, Inflammopharmacology aims to publish high-quality papers addressing these key problems and directed towards "advancing research into new medicines" as well as new approaches to development of safer and more effective, site-specific anti-inflammatory agents.

The prime focus of papers considered for inclusion in the journal is concentrated on the mechanisms of action and the use of anti-inflammatory agents in the treatment of rheumatic and related painful conditions. The journal also aims to publish papers on the fundamental and clinical aspects of the iatrogenic conditions that accompany treatment with many anti-rheumatic agents including those involving the gastro-intestinal, hepato-renal and cardiovascular systems. Papers concerned with the beneficial effects of anti-inflammatory drugs in the prevention of cancer, Alzheimer's and other neurodegenerative diseases are of particular interest for publication in this journal.

One of the central elements in understanding the modes of action of various anti-inflammatory agents is the recognition that drugs may be limited in their effectiveness, selectivity and toxicology as a consequence of the disease processes themselves. These "disease-drug interactions", can differ depending on the disease states being treated. This area of increasing interest being described as "conditional pharmacology", i.e. disease state(s) may influence drug actions and vice versa (see Inflammopharmacology 1991, 1:61; 1995, 3:363).

The widening use of NSAIDs in the treatment of neurodegenerative diseases, etc. reflects an obvious need for a multi-disciplinary journal, such as Inflammopharmacology.

The following diagram gives a perspective of scope and content of articles in Inflammopharmacology.
K. D. Rainsford $(\bowtie)$

Biomedical Research Centre, Sheffield Hallam University, Howard Street, Sheffield S1 1WB, UK

e-mail: k.d.rainsford@shu.ac.uk 


\section{Thematic Perspective of InflammoPharmacology}

'ADVANCING RESEARCH IN NEW MEDICINES'

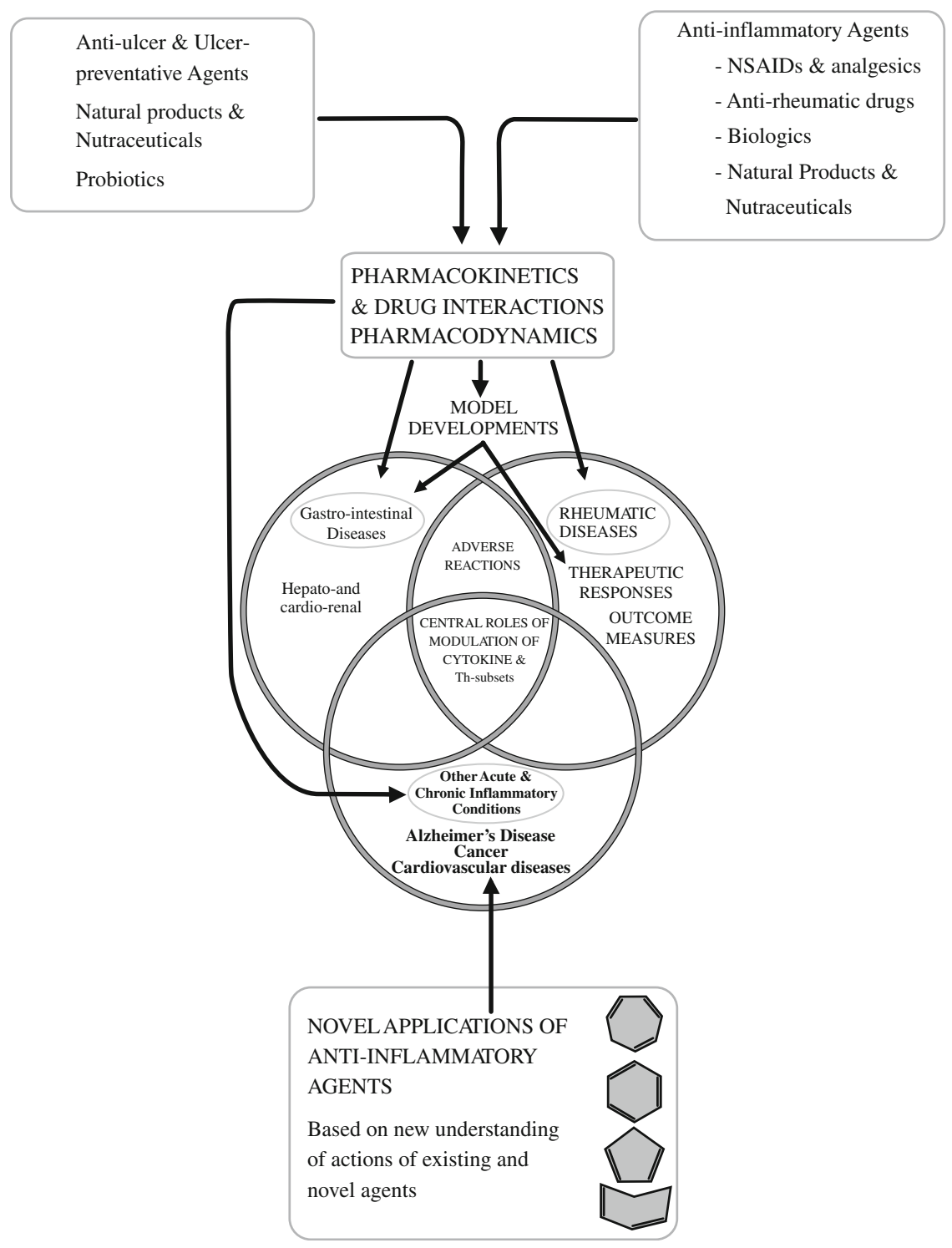

\section{(C) 2010 AK \& KD Rainsford}

The journal welcomes suggestions of other topics that might fall within its area of interest to widen both its attractiveness to authors and increase its readership.

K. D. Rainsford

Editor-in-Chief 